\title{
Nicotine exerts neuroprotective effects against $\beta$-amyloid-induced neurotoxicity in SH-SY5Y cells through the Erk1/2-p38-JNK-dependent signaling pathway
}

\author{
MAO QIANG XUE* ${ }^{*}$ XIAO XING LIU*, YAN LING ZHANG and FENG GUANG GAO \\ Department of Basic Medical Sciences, Medical College, Xiamen University, Xiamen, Fujian 361005, P.R. China
}

Received September 23, 2013; Accepted January 13, 2014

DOI: $10.3892 /$ ijmm.2014.1632

\begin{abstract}
Epidemiological data have indicated that smoking tobacco can decrease the risk of developing Alzheimer's disease (AD). Nicotine, a main component of tobacco, has been shown to have therapeutic effects in AD. The aim of the present study was to assess the neuroprotective effects of nicotine against toxicity induced by $\beta$-amyloid $(\mathrm{A} \beta)$ in relation to cell apoptosis, and to elucidate the role of the activation of the Erk1/2-p38-JNK pathway and the modulation of anti-apoptotic proteins in the nicotine-induced neuroprotective effects. We performed in vitro and in vivo experiments using SH-SY5Y cells and C57BL/6 mice, respectively. The effects of nicotine on cell apoptosis were determined by flow cytmetry and microscopic observation. The effects of nicotine on the expression of anti-apoptotic proteins were also determined by western blot analysis. Our results demonstrated that nicotine protected the SH-SY5Y cells against $\mathrm{A} \beta_{25-35}$-induced toxicity by inhibiting apoptosis and upregulating the expression of anti-apoptotic proteins. As shown by our in vivo experiments, nicotine effectively ameliorated the impairment in spatial working memory induced by $A \beta_{25-35}$; this was confirmed by a Morris water maze navigation test and further supported by the upregulation of Bcl-2 in the hippocampus of $\mathrm{A} \beta_{25-35}$-injected mice treated with nicotine. The phosphorylation of Erk1/2, p38 and JNK increased following treatment with nicotine in the SH-SY5Y cells, whereas caspase-3 activation was inhibited by treatment with nicotine prior to exposure to $\mathrm{A} \beta_{25-35}$. Of note, these effects of nicotine against $\mathrm{A} \beta_{25-35}$-induced damage were abolished by inhibitors of Erk1/2, p38 and JNK phosphorylation. These findings suggest that nicotine prevents $\mathrm{A} \beta_{25-35}$-induced neurotoxicity through the inhibition of neuronal
\end{abstract}

Correspondence to: Professor Feng Guang Gao or Professor Mao Qiang Xue, Department of Basic Medical Sciences, Medical College, Xiamen University, 168 Daxue Road, Xiamen, Fujian 361005, P.R. China

E-mail: gfengguang@xmu.edu.cn

E-mail: xmq8887@xmu.edu.cn

*Contributed equally

Key words: nicotine, Alzheimer's disease, Bcl-2, $\beta$-amyloid apoptosis, and may thus prove to be a potential preventive or therapeutic agent for AD.

\section{Introduction}

Alzheimer's disease (AD), the most common form of dementia in elder persons, is a neurodegenerative disease which is characterized by the progressive loss of memory, deterioration of language, as well as defects in visual and motor coordination (1). Documented data have shown that the pathological hallmarks of $\mathrm{AD}$ include the accumulation of intracellular neurofibrillary tangles rich in Tau protein and extracellular plaques containing $\beta$-amyloid protein $(A \beta)(2)$. Despite the fact that the precise cause of $A D$ remains unknown, toxic $A \beta$ accumulation-induced neuronal loss and $A \beta$, the constituent of extracellular plaques have been observed in patients with $\mathrm{AD}$. This indicates that $\mathrm{A} \beta$ may play an important role in the development of AD (3).

Oxidative stress, an imbalance toward the pro-oxidant side of pro-oxidant/antioxidant homeostasis, occurs in some brain neurodegenerative disorders. Although it has been reported that nicotine possibly promotes lung cancer development and reduces the efficacy of chemotherapeutic agents (4), the rapid synaptic transmission in key regions controlling behavior mediated by nicotine via nicotine acetylcholine receptors (nAChRs) has also reported (5). Notably, nicotine has been confirmed to improve memory function and reduce amyloid plaque burden in a transgenic mouse model of AD (6), which indicates that nicotine may be a survival agonist against apoptosis induced by various types of stress (7). However, the exact mechanisms of action of nicotine and its role in the improvement of $\mathrm{AD}$ remain unclear; elucidating these mechanisms pay prove useful in the treatment of $\mathrm{AD}$.

Bcl-2, Bcl-xL and Mcl-1, which belong to the Bcl-2 family, are localized in the outer mitochondrial membrane and protect cells against a variety of apoptotic stimuli (8). Despite the fact that nicotine increases Bcl-2, Bcl-xL and Mcl-1 expression and facilitates multiple drug resistance in lung cancer (9), the exact effects of the nicotine-induced upregulation of anti-apoptotic proteins in the treatment of AD have not yet been elucidated. Mitogen-activated protein kinase (MAPK) signaling pathways, which included Erk, SAPK/JNK and p38, play important roles in growth, differentiation, development and cell survival (10-12). Nicotine has been reported to activate the MAPK pathway in 
various tissues and cell types $(13,14)$. Other studies have also shown that the inhibition of MAPK pathways is involved in the anti-apoptotic effects and mediates neuroprotection (15). Hence, the exact roles of Erk, SAPK/JNK and p38 in nicotinemediated neuroprotection remain unelucidated.

In the present study, we aimed to investigate the protective effects of nicotine on $A \beta$-induced neurotoxicity. The effects of nicotine on A $\beta$-induced SH-SY5Y cell apoptosis were first determined by flow cytometry and microscopic observation. The effects of nicotine on Bcl-2, Bcl-xL and Mcl-1 expression, as well as MAPK kinase activation were further explored by western blot analysis. Using kinase inhibitors, the levels of Erk, SAPK/JNK and p38 phosphorylation, as well as caspase-3 activation, Bcl-xL expression and cell viability were further investigated. Importantly, the neuroprotective effects of nicotine on the impairment of spatial working memory by $A \beta_{25-35}$ and Bcl-2 expression were investigated by a Morris water maze navigation test and immunohistochemical analysis of hippocampal sections using a mouse model of $A \beta$-induced AD. The results revealed the following: firstly, $A \beta$ markedly augmented SH-SY5Y cell apoptosis, the release of cytochrome $c$ and caspase-3 activation in a time- and dose-dependent manner. Secondly, pre-treatment with nicotine attenuated the A $\beta$-induced cell apoptosis, inhibited caspase- 3 activation and increased cell viability. Thirdly, treatment with nicotine markedly upregulated Bcl-2, Bcl-xL and Mcl-1 expression and MAPK kinase phosphorylation. Notably, when the activities of Erk, SAPK/JNK and p38 were inhibited, the nicotine-induced Bcl-xL upregulation and anti-apoptotic effects were reversed accordingly. Importantly, in vivo nicotine administration greatly ameliorated the impairment in spatial working memory induced by $\mathrm{A} \beta_{25-35}$ and upregulated $\mathrm{Bcl}-2$ expression in the hippocampus. The data presented in this study indicate that nicotine exerts neuroprotective effects against $A \beta$-induced neurotoxicity by activating the MAPK pathway and upregulating the expression of anti-apoptotic proteins.

\section{Materials and methods}

Reagents. Nicotine and the $\mathrm{A} \beta_{25-35}$ peptide were purchased from Sigma-Aldrich (St. Louis, MO, USA). $A \beta_{25-35}$ was dissolved in deionized distilled water at a concentration of $5 \mathrm{mg} / \mathrm{ml}$. The stock solution was diluted to the desired concentrations immediately before use. Dulbecco's modified Eagle's medium (DMEM) and fetal bovine serum (FBS) were obtained from HyClone (Logan, USA). The Annexin V/PI Apoptosis detection kit was obtained from Promega (Madison, WI, USA). Erk1/2 inhibitor (U0126), p38 inhibitor (SB203580) and JNK inhibitor (SP600125), as well as antibodies to tubulin, Bcl-2, Bc-xL, Mcl-1, cleaved caspase-3, cytochrome $c$, phospho-p38, phosphoErk1/2, phospho-Mek1/2, phospho-p90Rsk, phospho-Msk, phospho-c-Jun, phospho-SAPK/JNK and phospho-MKK3/6 were purchased from Cell Signaling Technology Inc. (Beverly, MA, USA).

Animals. Pathogen-free C57BL/6 mice (male, 4 weeks old, weighing 18-22 g) were purchased from the Shanghai Laboratory Animal Center of the Chinese Academy of Sciences (Shanghai, China) and kept at the Animal Center of Xiamen University (Xiamen, China). All animal experiments were approved by the Review Board of the Medical College of Xiamen University.

Cell lines. Human SH-SY5Y neuroblastoma cells were obtained from the Shanghai Cell Bank (Shanghai, China). Cells were cultured in DMEM with $10 \% \mathrm{FBS}$ at $37^{\circ} \mathrm{C}$ in $5 \% \mathrm{CO}_{2}$ and passaged every 1-2 days to maintain logarithmic growth. Cells were synchronized by serum starvation for at least $12 \mathrm{~h}$ prior to treatment with nicotine or $A \beta_{25-35}$ for the indicated periods of time and concentrations.

Cell apoptosis assay. Cell apoptosis assay was determined by flow cytometry according to a previously described method (16). Briefly, 8x10 4 SH-SY5Y cells seeded in 24-well plates were pre-treated with $0.1 \mu \mathrm{M}$ nicotine and further treated with $A \beta_{25-35}$. The cells were then removed by trypsinization, rinsed with PBS and re-suspended in binding buffer containing Annexin V and propidium iodide (PI) for $20 \mathrm{~min}$ at room temperature. The samples were analyzed on a FACSCalibur flow cytometer and data were analyzed using CellQuest software.

Western blot analysis. Proteins were obtained in lysis buffer as previously described (13). Proteins were loaded onto SDS-PAGE gels for electrophoresis and transferred onto PVDF membranes. After blocking in 5\% fat-free milk in TBST for $90 \mathrm{~min}$, the membranes were incubated with primary antibodies at $4^{\circ} \mathrm{C}$ overnight. Subsequently, the membranes were incubated with corresponding horseradish peroxidase (HRP)conjugated secondary antibodies at room temperature for 90 min. After washing 6 times with TBST (for 10 min each), the bound antibodies were visualized using enhanced chemiluminescence (ECL). Tubulin was used as a loading control.

Grouping of mice and establishment of animal model of $A D$. To investigate the effects of nicotine on AD symptoms, a mouse model of AD induced by $\mathrm{A} \beta$ was established according to previously described method (17). Briefly, 4-week-old male C57BL/6 mice were randomly divided into the control group, the AD group and AD group pre-treated with nicotine $(n=7$ per group). The mice in the AD group pre-treated with nicotine were administered nicotine $(0.1125 \mathrm{mg} / \mathrm{kg})$ subcutaneously twice a day. Animals that were injected with the vehicle served as the controls. After 2 weeks of nicotine administration, the mice were anesthetized with $4 \%$ chloral hydrate and the scalps were incised and retracted to expose the skull. The lambda and bregma were aligned in the same horizontal plane. The AD group and AD group pre-treated with nicotine were injected with $5 \mu \mathrm{l}(5 \mathrm{mg} / \mathrm{ml}) \mathrm{A} \beta_{25-35}$ using a stereotaxic instrument (RWD Life Science Co., Ltd., Shenzhen, China) and a microinjector (Kd Scientific, Holliston, MA, USA) into the hippocampal CA1 region of the right hemisphere (coordinates: anteroposterior, $-2.0 \mathrm{~mm}$ from the bregma; lateral, $-2.0 \mathrm{~mm}$; dorsovental, $-3.5 \mathrm{~mm}$ ) with a $10 \mu \mathrm{l}$ Hamilton syringe driven by a microinjector at a speed of $1 \mu \mathrm{l} / \mathrm{min}$. After the injection, the needle was kept in the injection site for a further $10 \mathrm{~min}$ and then slowly withdrawn in $5 \mathrm{~min}$; simultaneously, mice in the control group were injected with identical doses of sodium chloride into the same area. On the 17 th day after the microinjection, the mice were subjected to a Morris water maze navigation test. On 
the 21st day after the microinjection, all mice in the 3 groups were firstly anesthetized, and then the mouse brain tissues were removed and fixed in $4 \%$ paraformaldehyde in phosphate buffer for $24 \mathrm{~h}$, and embedded in paraffin for H\&E staining, Congo red staining and immunohistochemical staining.

$H \& E$ staining and Congo red staining. Mice were sacrificed by $\mathrm{CO}_{2}$ asphyxiation, and the brains were fixed for $48 \mathrm{~h}$ in $4 \%$ paraformaldehyde in PBS. Free-floating sections $(50 \mu \mathrm{M})$ were obtained using a vibratome slicing system. Sections were deparaffinized with various concentrations of ethanol. For H\&E staining, the sections were stained with hematoxylin for $15 \mathrm{~min}$ and washed in running tap water for $20 \mathrm{~min}$. Counterstaining was performed with eosin. For Congo red staining, the sections were stained with methanol Congo red for 10-20 min and following by $0.2 \%$ alkaline ethanol staining. Finally, the sections were dehydrated in $95 \%$ and absolute alcohols for 2 changes of 2 min each and observed under a microscope.

Immunohistochemitry. Mice were sacrificed by $\mathrm{CO}_{2}$ asphyxiation, and the brains were fixed for $48 \mathrm{~h}$ in $4 \%$ paraformaldehyde in PBS. Free-floating sections $(50 \mu \mathrm{m})$ were obtained using a vibratome slicing system. Bcl-2 expression in the hippocampus was determined by immunohistochemitry according to a previously described method (18). Briefly, endogenous peroxidase activity was quenched for $30 \mathrm{~min}$ in $\mathrm{H}_{2} \mathrm{O}_{2}$, and the sections were subsequently incubated in $90 \%$ formic acid for $7 \mathrm{~min}$ to expose the epitope. The primary Bcl-2 antibody was applied, and the sections were incubated overnight at $4^{\circ} \mathrm{C}$. The sections were subsequently washed in TBS to remove the excess primary antibody. The sections were then incubated in biotinylated secondary antibody for $1 \mathrm{~h}$ at $20^{\circ} \mathrm{C}$. After a final wash of $20 \mathrm{~min}$, slides were developed with diaminobenzidine substrate by using the avidin-biotin HRP system (Vector Laboratories, Burlingame, CA, USA).

Morris water maze navigation test. To investigate the effects of nicotine administration on spatial learning and memory following exposure to $\mathrm{A} \beta$, the Morris water maze navigation test was performed according as previously described (19). Briefly, the water maze consisted of a circular water tank $\left(120 \mathrm{~cm}\right.$ in diameter) filled with water $\left(26^{\circ} \mathrm{C}\right)$ in which an escape platform $(10 \mathrm{~cm}$ in diameter) was hidden $0.5 \mathrm{~cm}$ below the surface of the 30 -cm-deep water. The water was made opaque by addition of milk powder, thereby rendering the platform invisible. A video camera was placed above the centre of the pool to capture images of the swimming animal. Mice were trained in a 4-trial per day task for 5 consecutive days. Each mouse was allowed a maximum of $60 \mathrm{sec}$ to find the hidden platform and allowed to remain on it for $15 \mathrm{sec}$. If a mouse failed to find the platform within $60 \mathrm{sec}$, the mouse was placed on the platform for $30 \mathrm{sec}$ by the investigator. The time required to find the hidden platform during these 4 acquisition trials was averaged. The navigation of the mice was tracked by a video camera. The escape latency of the mice was recorded. The experiment lasted 5 days, and each day was divided into morning and afternoon blocks, 4 trials in each block.

Statistical analysis. Each experiment was repeated at least 3 times and similar data were obtained. All data are presented as the means \pm standard error of the means (SEM). Statistical significance was examined using one-way or two-way ANOVA with a post hoc Newman-Keuls test. A value of $p<0.05$ was considered to indicate a statistically significant difference.

\section{Results}

$A \beta_{25-35}$ induces apoptosis of SH-SY5Y cells in a dose-and time-dependent manner. A $\beta$-induced neurotoxicity is thought to be a critical event in the pathogenesis of AD (2). In this study, to assess the neurotoxicity of $\mathrm{A} \beta, \mathrm{SH}-\mathrm{SY} 5 \mathrm{Y}$ cells were treated with $A \beta_{25-35}$ and cell viability was determined by flow cytometry, microscopic observation. Western blot analysis was also performed to determine the release of cytochrome $c$ and the levels of cleaved caspase-3. The results revealed that treatment with $\mathrm{A} \beta_{25-35}(20$ and $50 \mu \mathrm{M})$ induced approximately 7.27 and $13.8 \%$ SH-SY5Y cell apoptosis (Fig. 1A and B, p $<0.05$, $\mathrm{A} \beta_{25-35} 20 \mu \mathrm{M}$ vs. control; $\mathrm{p}<0.01, \mathrm{~A} \beta_{25-35} 50 \mu \mathrm{M}$ vs. control, one-way ANOVA with post hoc Newman-Keuls test) indicating that $\mathrm{A} \beta_{25-35}$ induced apoptosis in a dose-dependent manner. Microscopic observation also revealed similar results (Fig. 1C). Importantly, cleaved caspase- 3 and the release of cytochrome $c$ were increased following treatment with $A \beta_{25-35}$ in a timedependent manner (Fig. 1D). As the activation of caspases, in particular that of caspase-3, plays a prominent role in the initiation of apoptosis, the augmented cleaved caspase- 3 levels and the release of cytochrome $c$ induced by treatment with $A \beta_{25-35}$ indicated that $\mathrm{A} \beta$ exerts neurotoxic effects on $\mathrm{SH}-\mathrm{SY} 5 \mathrm{Y}$ cells and may contribute to the development of AD.

Pre-treatment with nicotine attenuates $A \beta_{25-35}$-induced neurotoxicity in $\mathrm{SH}-\mathrm{SY} 5 \mathrm{Y}$ cells. To explore the potential neuroprotective effects of nicotine against $A \beta$-induced neurotoxicity, the SH-SY5Y cells were treated with nicotine prior to $A \beta_{25-35}$ stimulation and cell apoptosis was determined by flow cytometry and microscopic observation. Western blot analysis was also performed to measure the levels of cleaved caspase- 3 . The results revealed that although treatment with $A \beta_{25-35}$ induced approximately 79\% SH-SY5Y cell apoptosis, pre-treatment with nicotine markedly abolished the neurotoxic effects of $\mathrm{A} \beta_{25-35}$ on SH-SY5Y cells (Fig. $2 \mathrm{~A}$ and $\mathrm{B}, \mathrm{p}<0.001, \mathrm{~A} \beta_{25-35}$ vs. control; $\mathrm{p}<0.001, \mathrm{~A} \beta_{25-35}$ vs. nicotine $0.01 \mu \mathrm{M} / \mathrm{A} \beta_{25-35} ; \mathrm{p}<0.001$, $\mathrm{A} \beta_{25-35}$ vs. nicotine $0.1 \mu \mathrm{M} / \mathrm{A} \beta_{25-35}$, one-way ANOVA with post hoc Newman-Keuls test). Microscopic observation also revealed similar results (Fig. 2C). Importantly, pre-treatment with nicotine markedly attenuated the $A \beta_{25-35}$-induced activation of caspase-3 (Fig. 2D). These data indicate that treatment with nicotine exerts marked neuroprotective effects against A $\beta$-induced neurotoxicity.

Nicotine increases Bcl-2, Bcl-xL and Mcl-1 expression in SH-SY5Y cells. Given that Bcl-2 family proteins are important modulators of cell apoptosis, we determined the effects of nicotine on Bcl-2, Bcl-xL and $\mathrm{Mcl}-1$ protein expression in the SH-SY5Y cells treated with $A \beta_{25-35}$ by western blot analysis. The results revealed that nicotine markedly increased Bcl-2, Bcl-xL and Mcl-1 expression in a dose- and time-dependent manner (Fig. 3). The results from western blot analysis (Fig. 3A) revealed that pre-treatment with nicotine $(0.01-100 \mu \mathrm{M}$ for $12 \mathrm{~h})$ markedly increased the Bcl-2, Bcl-xL and Mcl-1 protein levels in 
A
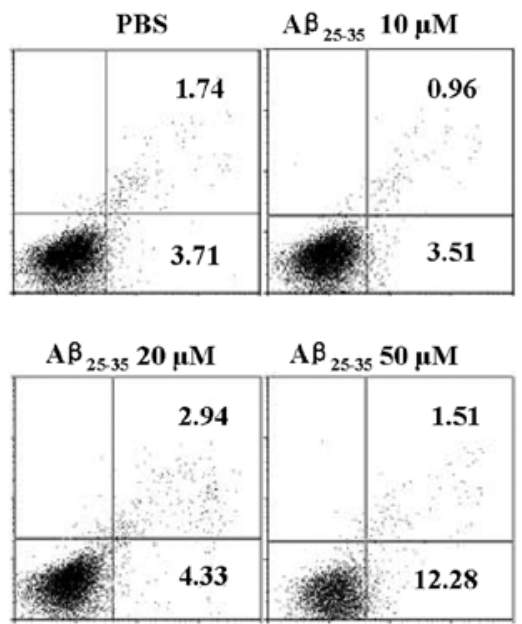

C
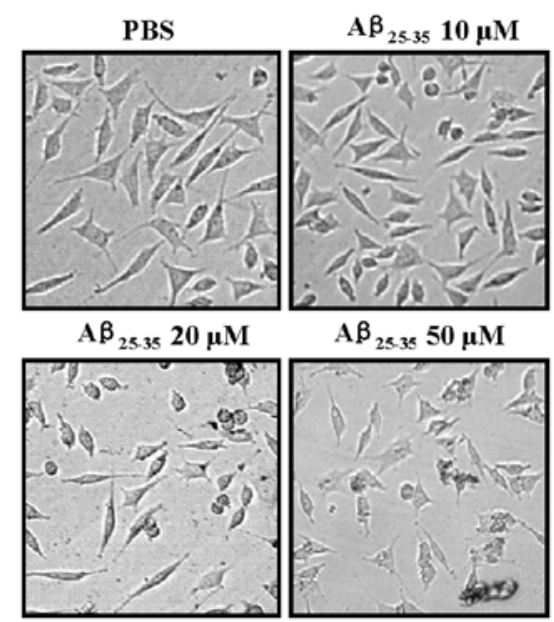

B

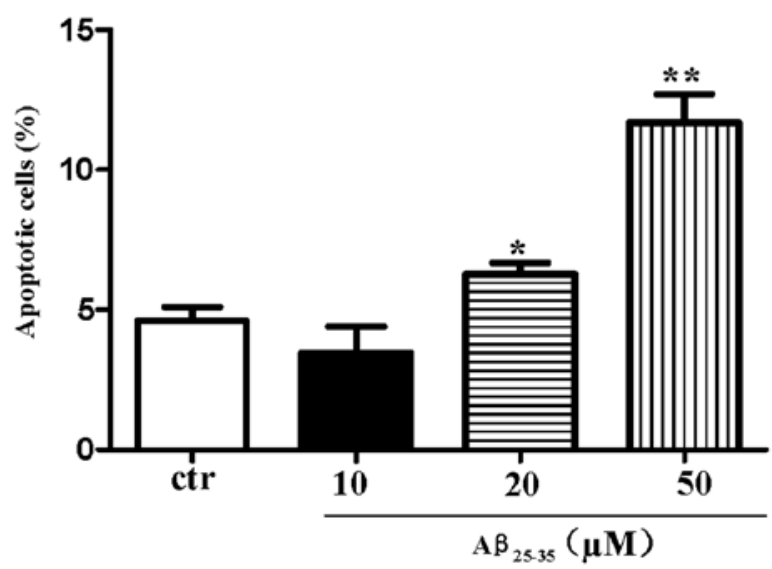

D

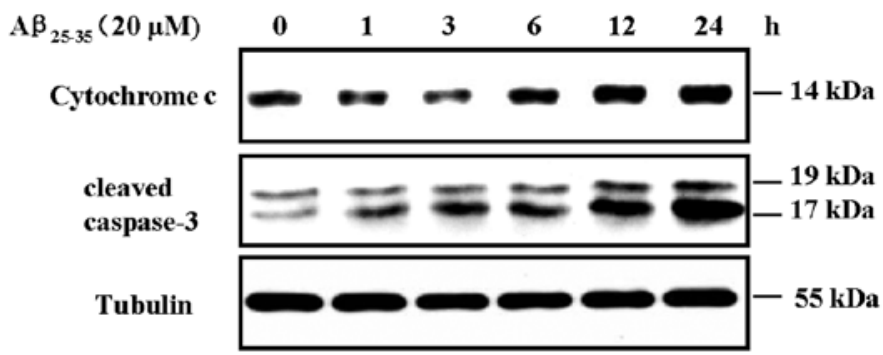

Figure 1. A $\beta_{25-35}$ induces apoptosis in SH-SY5Y cells. To investigate the effects of A $\beta$ on cell apoptosis, SH-SY5Y cells were pre-treated with 10-50 $\mu \mathrm{M} A \beta_{25-35}$ for $24 \mathrm{~h}$ and cell viability was determined by (A and B) flow cytometry; (C) microscopic observation; and (D) western blot analysis. (A) The percentage of apoptotic cells was determined by flow cytometry with Annexin V and PI staining. The numbers in the histogram indicate the percentage of apoptotic cells. (B) Bar graph of results in (A). Data are presented as the means \pm SEM. A $\beta_{25-35} 20 \mu \mathrm{M}$ vs. control, ${ }^{*} \mathrm{p}<0.05 ; \mathrm{A} \beta_{25-35} 50 \mu \mathrm{M}$ vs. control, ${ }^{* *}$ p $<0.001$. One-way ANOVA with post hoc Newman-Keuls test. (C) Cell morphology was observed under a microscope. (D) $\mathrm{A} \beta_{25-35}$ treatment increased the release of cytochrome $c$ and caspase-3 activation. Tubulin was used as an internal control. A representative out of 3 independent experiments is shown.

the SH-SY5Y cells. Pre-treatment with $0.1 \mu \mathrm{M}$ nicotine induced the most marked upregulation of Bcl-2, Bcl-xL and Mcl-1 expression. Treatment with $0.1 \mu \mathrm{M}$ nicotine increased Bcl-2, Bcl-xL and Mcl-1 expression in a time-dependent manner. As the Bcl-2 family play an important role in regulating apoptosis, as well as the mitochondrial-initiated release of cytochrome $c$ and activation of caspases (20), the fact that nicotine attenuated the A $\beta$-induced activation of caspase-3, indicated that nicotine may affect Bcl-2 family protein expression (21).

Nicotine increases Erk1/2, p38 and SAPK/JNK phosphorylation in SH-SY5Y cells. The Erk1/2, p38 and SAPK/JNK pathways are involved in regulating the expression of antiapoptotic proteins (22-24). To investigate the role of the Erk1/2, p38 and SAPK/JNK pathways in the nicotine-induced increase in Bcl-2, Bcl-xL and Mcl-1 expression, the effects of nicotine on Erk1/2, p38 and SAPK/JNK MAPK kinase activation were determined. In initial experiments, SH-SY5Y cells were stimulated with a range of nicotine concentrations $(0.01-100 \mu \mathrm{M})$ for 5 min, and the phosphorylation levels of Erk1/2,p38 and c-Jun MAPKs were determined by western blot analysis. Nicotine
(0.1-100 $\mu \mathrm{M})$ induced a significant enhancement of Erk1/2, p38 and c-Jun MAPK phosphorylation in a dose-dependent manner (Fig. 4A, C and E), approximately $100 \%$ above basal levels. Importantly, Mek1/2, p90Rsk, Msk1, SAPK/JNK and MKK3/6 were also activated following treatment with nicotine (Fig. 4A, $\mathrm{C}$ and $\mathrm{E})$.

The time course of the activation of Erk1/2, p38 and c-Jun MAPKs by $0.1 \mu \mathrm{M}$ nicotine in the SH-SY5Y cells indicated that Erk1/2, p38 and c-Jun MAPK phosphorylation was increased at $5 \mathrm{~min}$ and persisted over $30 \mathrm{~min}$ of nicotine stimulation (Fig. 4B, D and F). Importantly, MeK1/2, p90Rsk, Msk1, SAPK/JNK and MKK3/6 were activated at 5 to $30 \mathrm{~min}$ following treatment with $0.1 \mu \mathrm{M}$ nicotine (Fig. $4 \mathrm{~B}, \mathrm{D}$ and $\mathrm{F}$ ). These results indicate that nicotine activates MAPK pathways and this may play an important role in the nicotine-mediated induction of anti-apoptotic proteins.

Nicotine upregulates Bcl-xL expression by activating the Erk1/2, p38 and SAPK/JNK pathways. To explore the role of nicotine-activated MAPK pathways in the nicotine-induced increase in the expression of anti-apoptotic proteins, the 
A

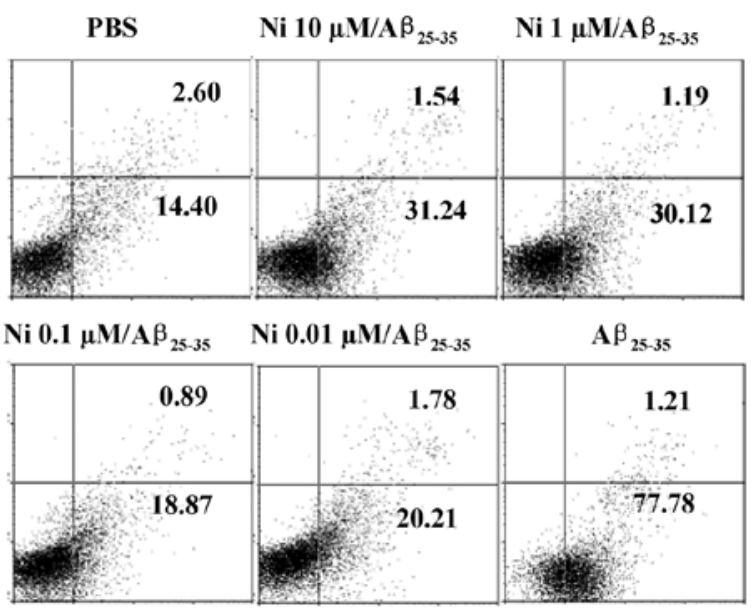

B

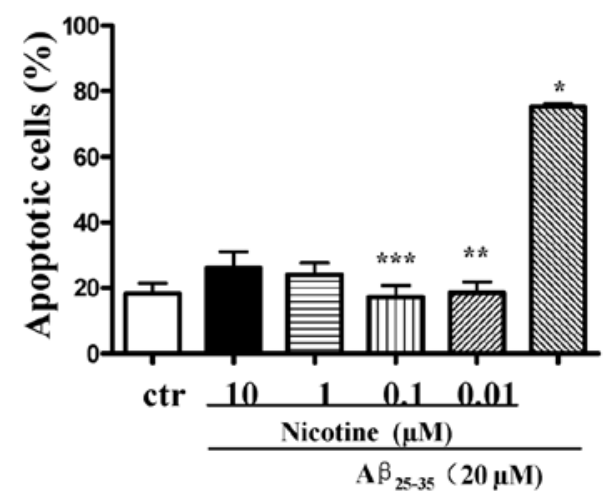

C
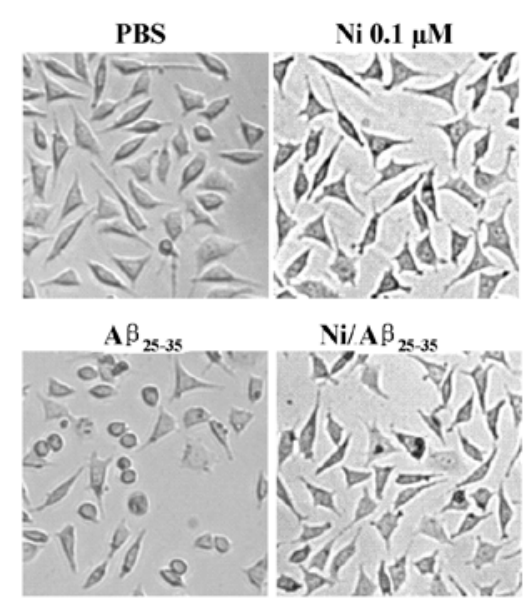

D

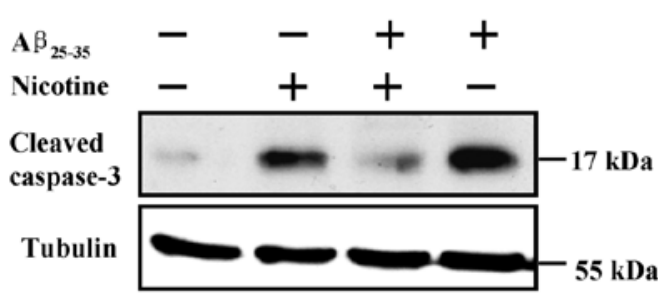

Figure 2. Prevention of $A \beta_{25-35}$-induced cell death by nicotine. To investigate the neuroprotective effects of nicotine against $\mathrm{A} \beta$-induced apoptosis, SH-SY5Y cells were treated with nicotine (0.01-10 $\mu \mathrm{M})$ prior to $20 \mu \mathrm{M} \mathrm{A} \beta_{25-35}$ stimulation for $24 \mathrm{~h}$ and cell apoptosis was determined by (A and B) flow cytometry; (C) microscopic observation and (D) western blot analysis. (A) The percentage of apoptotic cells were determined by flow cytometry with Annexin V and PI staining. The numbers in histogram indicate the percentage of apoptotic cells. (B) Bar graph of results in (A). Data shown are the means \pm SEM. A $\beta_{25-35} 20 \mu \mathrm{M}$ vs. control, ${ }^{*} \mathrm{p}<0.001 ; \mathrm{A} \beta_{25-35} 20 \mu \mathrm{M}$ vs. nicotine $(0.01 \mu \mathrm{M}) / \mathrm{A} \beta_{25-35} 20 \mu \mathrm{M},{ }^{* *} \mathrm{p}<0.001 ; \mathrm{A} \beta_{25-35} 20 \mu \mathrm{M}$ vs. nicotine $(0.1 \mu \mathrm{M}) / \mathrm{A} \beta_{25-35} 20 \mu \mathrm{M},{ }^{* * *} \mathrm{p}<0.001$. One-way ANOVA with post hoc Newman-Keuls test. (C) Cell morphology was observed under a microscope. (D) Nicotine treatment abrogated A $\beta_{25-35}$-induced caspase-3 activation. Tubulin was used as an internal control. A representative out of 3 independent experiments is shown. Ni, nicotine.

A

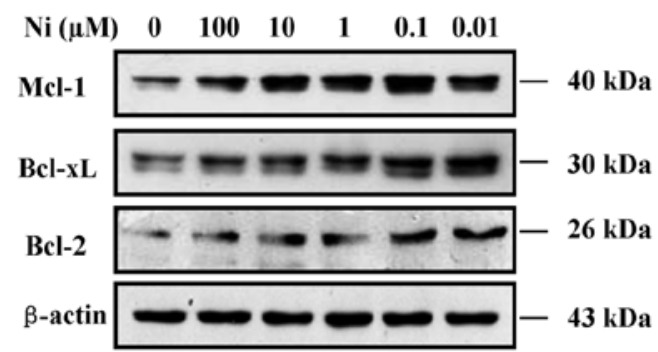

B

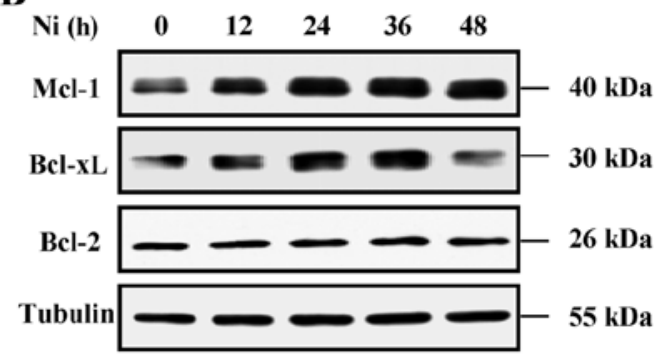

Figure 3. Nicotine treatment increases Bcl-2, Bcl-xL and Mcl-1 expression. To explore the effects of nicotine on Bcl-2, Bcl-xL and Mcl-1 expression, SH-SY5Y cells were treated with (A) various concentrations of nicotine for $12 \mathrm{~h}$ or (B) $0.1 \mu \mathrm{M}$ nicotine for the indicated periods of time. Whole cellular protein was extracted and Bcl-2, Bcl-xL and Mcl-1 expression was determined by western blot analysis. A representative out of 3 independent experiments is shown. Tubulin was used as an internal control.

SH-SY5Y cells were treated with inhibitors of related kinases and the expression of $\mathrm{Bcl}-2 / \mathrm{Bcl}-\mathrm{xL}$ and the activation of caspase- 3 were determined by western blot analysis. The results revealed that while pre-treatment with nicotine attenuated the A $\beta$-induced caspase- 3 activation, treatment with inhibitors of Erk1/2, p38 and SAPK/JNK markedly promoted the activation of caspase-3. The results also revealed that the nicotine-induced increase in Bcl-xL expression was dependent on Erk1/2 (U0126), p38 (SB203580) and SAPK/JNK (SP600125) phosphorylation (Fig. 5A). Notably, SAPK/JNK, but not Erk1/2 or $\mathrm{p} 38$, was shown to play a more important role in the attenuation of $\mathrm{A} \beta_{25-35}$-induced caspase-3 activation and $\mathrm{Bcl}-\mathrm{xL}$ expression (Fig. 5A). Microscopic observation also revealed similar results (Fig. 5B). These data indicate that the Erk1/2, p38 and SAPK/ 
A

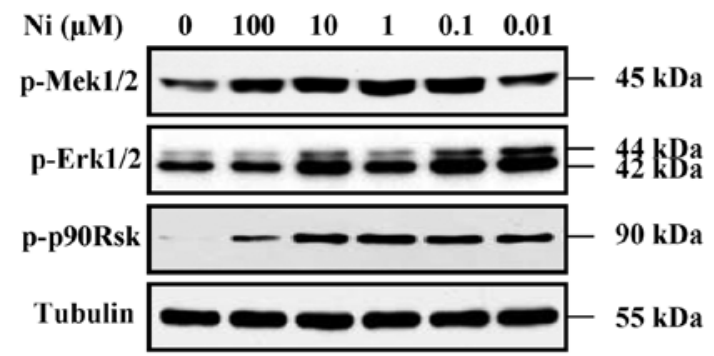

C

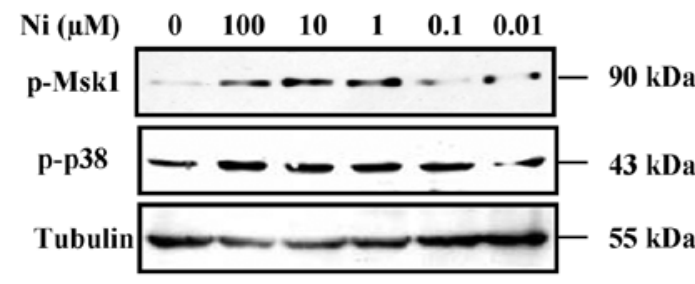

E

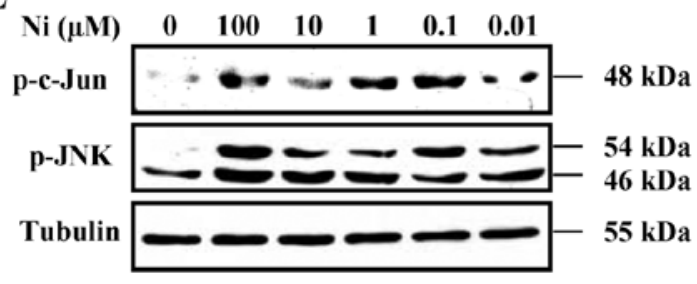

B

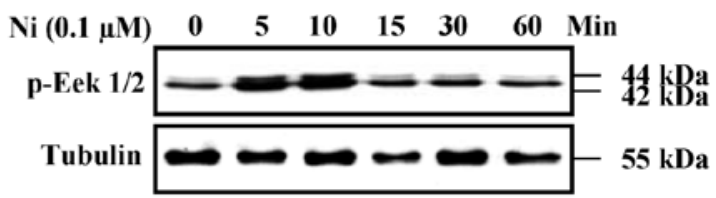

D

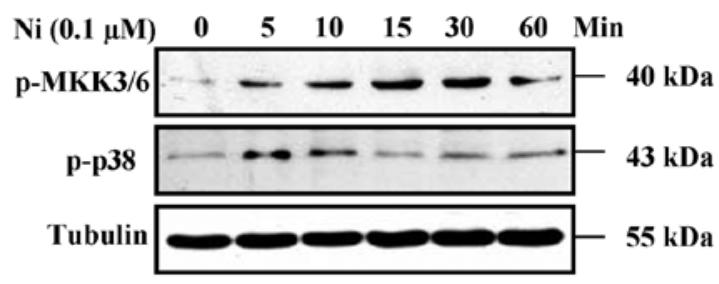

F

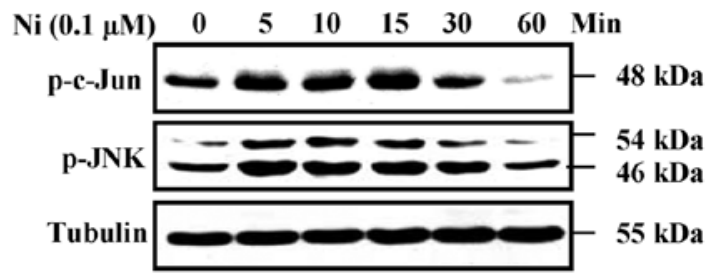

Figure 4. Nicotine treatment activates the Erk1/2, p38 and SAPK/JNK signaling pathways. To investigate the effects of nicotine on MAPK kinase activation, SH-SY5Y cells were treated with nicotine (0.1-100 $\mu \mathrm{M}$ ) for $10 \mathrm{~min}$ (A, C and E), or $0.1 \mu \mathrm{M}$ nicotine for the indicated periods of time (B, D and F). Whole cellular protein was extracted and the phosphorylation of MAPK kinases was determined by western blot analysis. A representative out of 3 independent experiments is shown. Tubulin was used as an internal control.

A

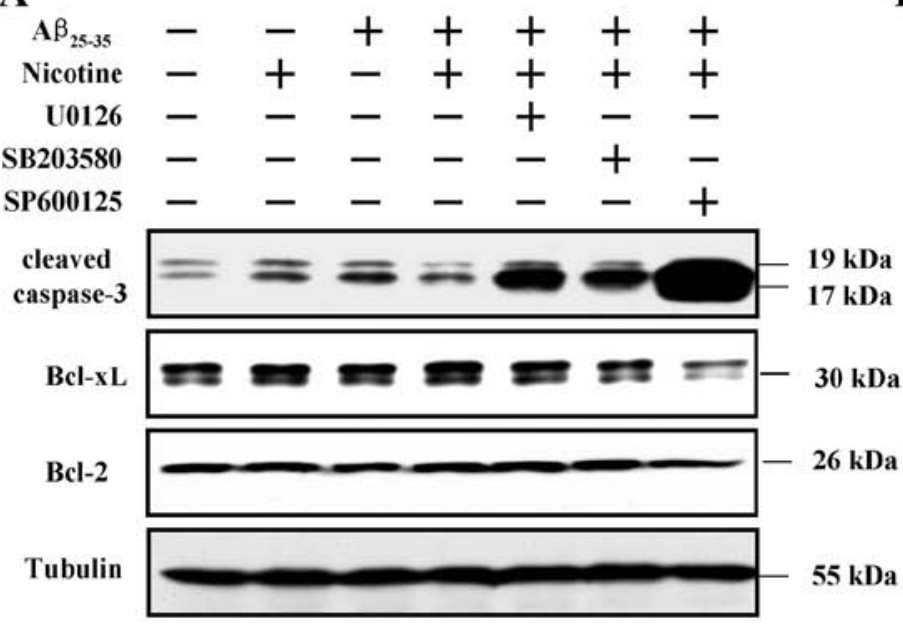

B
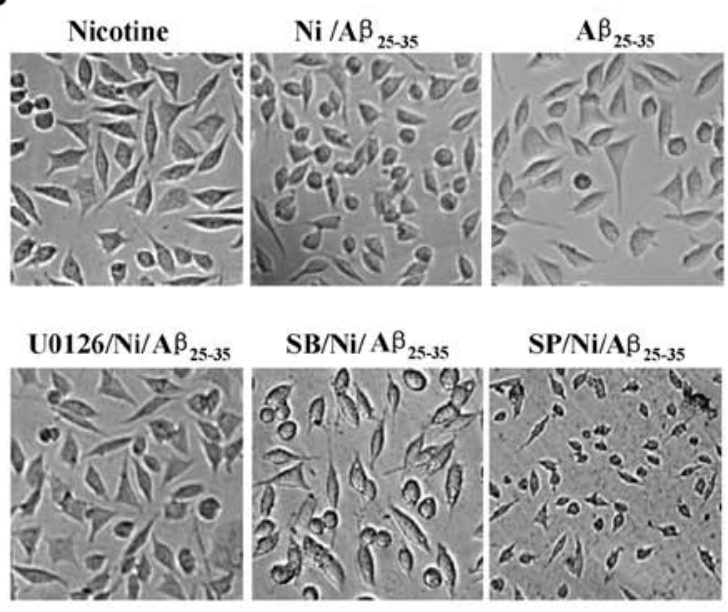

Figure 5. Erk, 338 and SARK/JNK pathways are involved in the nicotine-mediated neuroprotective effects against A $\beta_{25-35}$-induced neurotoxicity. To explore the role of the Erk, p38 and SARK/JNK pathways in the nicotine mediated neuroprotective effects, SH-SY5Y cells were treated with $10 \mu \mathrm{M}$ U0126 (Erk inhibitor), SB203580 (p38 inhibitor) or SP600125 (JNK inhibitor) prior to treatment with $0.1 \mu \mathrm{M}$ nicotine and treatment with $20 \mu \mathrm{M}$ A $\beta_{25-35}$. The expression of $\mathrm{Bcl}-2 / \mathrm{Bcl}-\mathrm{xL}$ and cell viability were determined by (A) western blot analysis and (B) microscopic observation, respectively. Tubulin was used as an internal control. A representative out of 3 independent experiments is shown. Ni:nicotine; SB, SB203580; SP, SP600125.

JNK pathways, particularly SAPK/JNK, play an important role in the nicotine-mediated anti-apoptotic effects.

Treatment with nicotine markedly ameliorates cognitive deficits in mice injected with $A \beta_{25-35}$. To investigate the effects of nicotine on spatial learning deficits induced by $A \beta_{25-35}$ injection, C57BL/6 mice were administered nicotine prior to the $A \beta_{25-35}$ injection and the ability of the mice to learn and process spatial information was examined by a Morris water maze. In the hidden platform test, the mice became more efficient at finding the platform on a successive trail (Fig. 6A). Two-way ANOVA (3 groups, 5 days) with repeated measures 
A

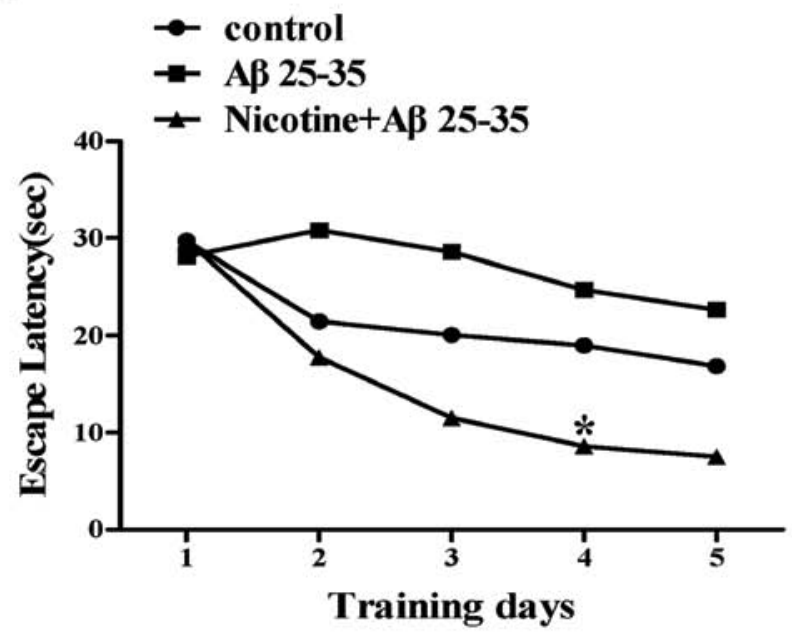

B

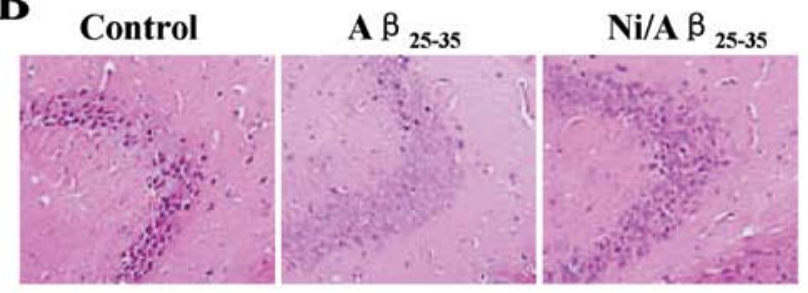

C

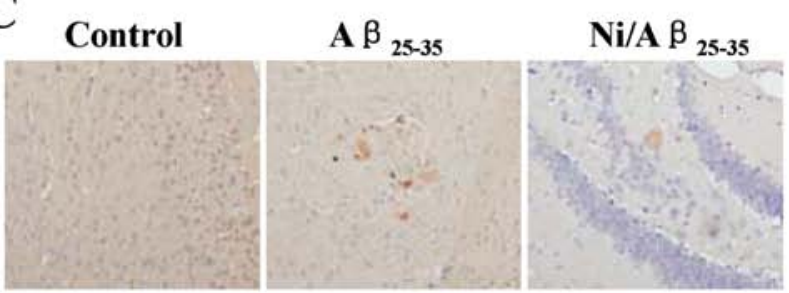

D

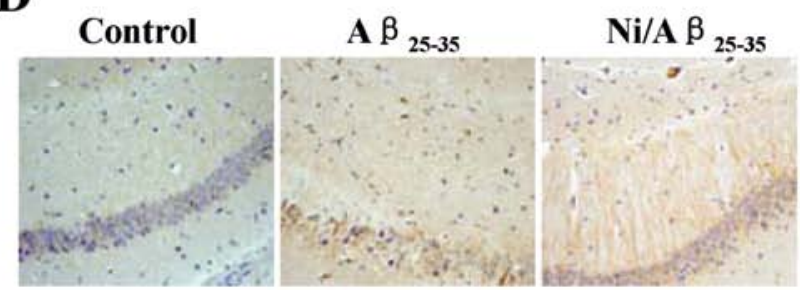

Figure 6. Nicotine attenuates $A \beta_{25-35}$-induced spatial working memory impairment by upregulating Bcl-2 expression. To investigate the effects of nicotine on spatial learning deficits induced by the $A \beta_{25-35}$-injection, $C 57 \mathrm{BL} / 6$ mice $(\mathrm{n}=7)$ were subcutaneously administered $0.1125 \mathrm{mg} / \mathrm{kg}$ nicotine twice a day for 2 weeks prior to $4.0 \mu \mathrm{g}$ hippocampal $\mathrm{A} \beta_{25-35}$ injection. The ability of the mice to learn and process spatial information was examined by performing a Morris water maze test $(\mathrm{A})$ on the 17 th day after the $\mathrm{A} \beta_{25-35}$ injection. On the 21st day after the $A \beta_{25-35}$ injection, all the mice were sacrificed and brain tissues were fixed in $4 \%$ paraformaldehyde. Animals that were injected with saline served as the vehicle controls. Free-floating sections $(50 \mu \mathrm{M})$ were obtained using a vibratome slicing system and subjected to (B) H\&E staining, (C) Congo red staining or (D) immunohistochemitry. (A) Nicotine administration attenuated the $\mathrm{A} \beta_{25-35}$-induced impairment in spatial working memory. Data shown are the means \pm SEM, $n=7$. " $\mathrm{p}<0.01$, two-way ANOVA. (B) Cell morphology was investigated by $\mathrm{H} \& \mathrm{E}$ staining. (C) Nicotine pre-treatment attenuated $\mathrm{A} \beta$ deposition in the hippocampal region. (D) In vivo nicotine administration augmented Bcl-2 expression in the hippocampal region. $\mathrm{Ni}$, nicotine.

rendered that the effect were statistically significant $(\mathrm{p}<0.01)$. Post hoc analysis (Student-Newman-Keuls) revealed that the
$\mathrm{A} \beta_{25-35}$-injected mice had a significantly longer escape latency (took longer to find the platform) than the sham-operated mice on days 2-5; a marked decrease in escape latency was observed following the administration of nicotine as compared with the $\mathrm{A} \beta_{25-35}$-injected mice (Fig. 6A), indicating that the injection of $A \beta_{25-35}$ greatly impaired spatial working memory and that treatment with nicotine attenuated this impairment in memory.

To investigate the effects of nicotine on cell viability in $\mathrm{A} \beta_{25-35}$-injected mice, $\mathrm{A} \beta$ accumulation and $\mathrm{Bcl}-2$ expression, hippocampal tissue sections from the mice were stained with $\mathrm{H} \& \mathrm{E}$ and Congo red, and immunohistochemitry was also performed. The results revealed that despite the fact that the $\mathrm{A} \beta_{25-35}$ injection markedly decreased cell viability (Fig. 6B) and increased $A \beta$ deposition (Fig. 6C), nicotine administration greatly attenuated $A \beta$ accumulation and abolished the $\mathrm{A} \beta_{25-35}$-induced neurotoxicity (Fig. 6B and C). Bcl-2 immunohistochemitry indicated that pre-treatment with nicotine markedly upregulated Bcl-2 expression (Fig. 6D). These results indicate that the neuroprotective effects of nicotine on $\mathrm{A} \mathrm{\beta}_{25-35^{-}}$ induced neurotoxicity may be mediated by the upregulation in the expression of anti-apoptotic proteins, such as Bcl-2.

\section{Discussion}

In recent studies, we investigated the biological role of nicotine and found that nicotine activates bone marrow-derived dendritic cells and that nicotine exerts potential anti-tumor effects on dendritic cells $(13,14,25)$. Nicotine has also been shown to mediate synaptic transmission in regions controlling behavior (5) and to improve memory function in a transgenic mouse model of $\mathrm{AD}(6)$, indicating that nicotine may be a potential therapeutic agent in AD. In the present study, JNK and $\alpha 7 \mathrm{nAchR}$ were found to be potential molecules in the treatment of AD. Firstly, the $A \beta$-induced increase in cell apoptosis, caspase- 3 activation and the release of cytochrome $c$ were markedly abrogated by treatment with nicotine. Secondly, the upregulation in Bcl-2, Bcl-xL and Mcl-1 and MAPK kinase phosphorylation was achieved following treatment with nicotine. Of note, the inhibition of MAPK activity markedly promoted caspase-3 activation and the downregulated nicotine-induced increase in Bcl-xL expression; importantly, nicotine administration improvement memory function and reduced $A \beta$ accumulation in a mouse model of $A \beta$-induced AD.

MAPK signaling pathways, which include Erk, SAPK/JNK and p38, play important roles in cell survival (10-12,22-24). The Erk 1/2 signaling pathway has been found to be involved in nicotine-mediated neuroprotection in spinal cord neurons (26). The p38/JNK MAPK pathway mediates cortical neuronal apoptosis (27). In the present study, using Erk1/2, p38 and JNK kinase inhibitors, the inhibition of Erk1/2, p38 and JNK kinases markedly increased caspase- 3 activation and abrogated the nicotine-mediated Bcl-xL upregulation (Fig. 5A), indicating that nicotine increased $\mathrm{Bcl}-\mathrm{xL}$ expression and attenuated $\mathrm{A} \beta$-induced neurotoxicity by activating the Erk1/2, p38 and JNK pathways in SH-SY5Y cells. The PI3K/Akt and NF- $\kappa \mathrm{B}$ pathways are also involved in nicotine-mediated neuroprotection $(28,29)$. However, the exact role of the PI3K-Akt and $\mathrm{NF}-\kappa \mathrm{B}$ pathways in nicotine-mediated neuroprotective effects against $A \beta$ stimulation remain unknown and require further investigation. 
JNK/SAPK, one of the vital signal transduction pathways which transmits and converts stress signaling into apoptotis signaling (27), has been reported to induce receptormediated apoptosis by upregulating Fas ligand expression in $\mathrm{T}$ lymphocytes (30) and is necessary for irradiation-induced mitochondrial-mediated apoptosis in embryonic fibroblasts (31). JNK activation has an anti-apoptotic function in cardiac myocytes (32). In the present studys, the inhibition of JNK kinase increased the activity of caspase-3 and downregulated Bcl-xL (Fig. 5A), indicating that JNK phosphorylation facilitates the survival of SH-SY5Y cells. The anti-apoptotic function of JNK may be due to the nicotine-induced JNK activation and may be cell type-dependent, as JNK has been found to play either a pro-apoptotic or anti-apoptotic role depending on cell type (31).

Bcl-2, downstream of the SAPK/JNK cascade, plays important roles in regulating programmed cell death and has been shown to be associated with neurodegenerative disorders, including $\mathrm{AD}$ (33). In the present study, both JNK activation (Fig. 4) and $\mathrm{Bcl}-2$ upregulation augmented by nicotine treatment were observed. As Bcl-2 is located in the outer mitochondrial membrane and protects cells against a variety of apoptotic stimuli (34), the nicotine-mediated Bcl-2 upregulation (Fig. 3) and anti-apoptotic effects (Fig. 2) indicated that the $A \beta$-induced apoptosis was mitochondrial-dependent. Hence, it was not surprising to find that $A \beta$ treatment mrakedly promoted the release of cytochrome $c$ and mitochondrialdependent caspase-3 activation (Fig. 1). As nicotine treatment significantly augmented Bcl-xL and Mcl-1 expression, the exact roles of these proteins in the nicotine-mediated antiapoptotic effects require further investigation.

Taken together, our data reveal that the anti-apoptotic effects of nicotine may be mediated by the increased expression of Bcl-2 family proteins and MAPK kinase phosphorylation, indicating that JNK may be a potential molecule in the treatment of AD.

\section{Acknowledgements}

We thank Jin Hua Su and Fu Chen for providing excellent animal care. This study was supported by grants from the National Natural Science Foundation of China (no. 81273203) and the Natural Science Foundation of Xiamen (no. 3502Z20104002).

\section{References}

1. Chan KY, Wang W, Wu JJ, Liu L, Theodoratou E, Car J, Middleton L, Russ TC, Deary IJ, Campbell H, Wang W and Rudan I: Epidemiology of Alzheimer's disease and other forms of dementia in China, 1990-2010: a systematic review and analysis. Lancet 381: 2016-2023, 2013.

2. Rosenberg RN and Lambracht-Washington D: DNA A $\beta 42$ vaccination as possible alternative immunotherapy for Alzheimer disease. JAMA Neurol 70: 772-773, 2013.

3. Nalivaeva NN and Turner AJ: The amyloid precursor protein: a biochemical enigma in brain development, function and disease. FEBS Lett 587: 2046-2054, 2013.

4. Tournier JM and Birembaut P: Nicotinic acetylcholine receptors and predisposition to lung cancer. Curr Opin Oncol 23: 83-87, 2011.

5. Albuquerque EX, Pereira EF, Alkondon M and Rogers SW: Mammalian nicotinic acetylcholine receptors: from structure to function. Physiol Rev 89: 73-120, 2009.
6. Levin ED, McClernon FJ and Rezvani AH: Nicotinic effects on cognitive function: behavioral characterization, pharmacological specification, and anatomic localization. Psychopharmacology 184: 523-539, 2006.

7. Cardinale A, Nastrucci C, Cesario A and Russo P: Nicotine: specific role in angiogenesis, proliferation and apoptosis. Crit Rev Toxicol 42: 68-89, 2012.

8. Kale J, Liu Q, Leber B and Andrews DW: Shedding light on apoptosis at subcellular membranes. Cell 151: 1179-1184, 2012.

9. Ke SZ, Ni XY, Zhang YH, Wang YN, Wu B and Gao FG: Camptothecin and cisplatin upregulate ABCG2 and MRP2 expression by activating the ATM/NF- $\kappa \mathrm{B}$ pathway in lung cancer cells. Int J Oncol 42: 1289-1296, 2013.

10. Sweatt JD: The neuronal MAP kinase cascade: a biochemical signal integration system subserving synaptic plasticity and memory. J Neurochem 76: 1-10, 2001.

11. Thornton TM and Rincon M: Non-classical p38 map kinase functions: cell cycle checkpoints and survival. Int J Biol Sci 5: 44-51, 2009.

12. Zhuang S and Schnellmann RG: A death-promoting role for extracellular signal-regulated kinase. J Pharmacol Exp Ther 319: 991-997, 2006.

13. Jin HJ, Li HT, Sui HX, Xue MQ, Wang YN, Wang JX and Gao FG: Nicotine stimulated bone marrow-derived dendritic cells could augment HBV specific CTL priming by activating PI3K-Akt pathway. Immunol Lett 146: 40-49, 2012.

14. Jin HJ, Sui HX, Wang YN and Gao FG: Nicotine up-regulated 4-1BBL expression by activating Mek-PI3K pathway augments the efficacy of bone marrow-derived dendritic cell vaccination. $J$ Clin Immunol 33: 246-254, 2013.

15. Dineley KT, Westerman M, Bui D, Bell K, Ashe KH and Sweatt JD: Beta-amyloid activates the mitogen-activated protein kinase cascade via hippocampal alpha7 nicotinic acetylcholine receptors: In vitro and in vivo mechanisms related to Alzheimer's disease. J Neurosci 21: 4125-4133, 2001.

16. Hu SX, Sui HX, Jin HJ, Ni XY, Liu XX, Xue MQ, Zhang Y and Gao FG: Lipopolysaccharide and dose of nicotine determine the effects of nicotine on murine bone marrow-derived dendritic cells. Mol Med Rep 5: 1005-1010, 2012.

17. Oddo S, Caccamo A, Green KN, Liang K, Tran L, Chen Y, Leslie FM and LaFerla FM: Chronic nicotine administration exacerbates tau pathology in a transgenic model of Alzheimer's disease. Proc Natl Acad Sci USA 102: 3046-3051, 2005.

18. Zhang J, Liu Q, Chen Q, Liu NQ, Li FL, Lu ZB, Qin C, Zhu H, Huang YY, He W and Zhao BL: Nicotine attenuates betaamyloid-induced neurotoxicity by regulating metal homeostasis. FASEB J 20: 1212-1214, 2006.

19. Li J, Wang G, Liu J, Zhou L, Dong M, Wang R, Li X, Li X, Lin C and Niu Y: Puerarin attenuates amyloid-beta-induced cognitive impairment through suppression of apoptosis in rat hippocampus in vivo. Eur J Pharmacol 649: 195-201, 2010.

20. Plotnikov EY, Morosanova MA, Pevzner IB, Zorova LD, Manskikh VN, Pulkova NV, Galkina SI, Skulachev VP and Zorov DB: Protective effect of mitochondria-targeted antioxidants in an acute bacterial infection. Proc Natl Acad Sci USA 110: E3100-E3108, 2013.

21. Cui WY, Wang J, Wei J, Cao J, Chang SL, Gu J and Li MD: Modulation of innate immune-related pathways in nicotinetreated SH-SY5Y cells. Amino Acids 43: 1157-1169, 2012.

22. Park HY, Kim GY, Kwon TK, Hwang HJ, Kim ND, Yoo YH and Choi YH: Apoptosis induction of human leukemia U937 cells by 7,8-dihydroxyflavone hydrate through modulation of the Bcl-2 family of proteins and the MAPKs signaling pathway. Mutat Res 751: 101-108, 2013.

23. Zhu X, Jiang Y, Shan PF, Shen J, Liang QH, Cui RR, Liu Y, Liu GY, Wu SS, Lu Q, Xie H, Liu YS, Yuan LQ and Liao EY: Vaspin attenuates the apoptosis of human osteoblasts through ERK signaling pathway. Amino Acids 44: 961-968, 2013.

24. Chi J, Zhu Y, Fu Y, Liu Y, Zhang X, Han L, Yin X and Zhao D: Cyclosporin A induces apoptosis in H9c2 cardiomyoblast cells through calcium-sensing receptor-mediated activation of the ERK MAPK and p38 MAPK pathways. Mol Cell Biochem 367: 227-236, 2012.

25. Gao FG, Wan DF and Gu JR: Ex vivo nicotine stimulation augments the efficacy of therapeutic bone marrow-derived dendritic cell vaccination. Clin Cancer Res 13: 3706-3712, 2007.

26. Toborek M, Son KW, Pudelko A, King-Pospisil K, Wylegala E and Malecki A: ERK 1/2 signaling pathway is involved in nicotine-mediated neuroprotection in spinal cord neurons. J Cell Biochem 100: 279-292, 2007. 
27. Li Y, Chen G, Zhao J, Nie X, Wan C, Liu J, Duan Z and $\mathrm{Xu}$ G: 2,3,7,8-Tetrachlorodibenzo-p-dioxin (TCDD) induces microglial nitric oxide production and subsequent rat primary cortical neuron apoptosis through p38/JNK MAPK pathway. Toxicology 312: 132-141, 2013.

28. Huang X, Cheng Z, Su Q, Zhu X, Wang Q, Chen R and Wang X Neuroprotection by nicotine against colchicine-induced apoptosis is mediated by PI3-kinase - Akt pathways. Int J Neurosci 122: 324-332, 2012.

29. Barr J, Sharma CS, Sarkar S, Wise K, Dong L, Periyakaruppan A and Ramesh GT: Nicotine induces oxidative stress and activates nuclear transcription factor kappa B in rat mesencephalic cells. Mol Cell Biochem 297: 93-99, 2007.

30. Davis RJ: Signal transduction by the JNK group of MAP kinases. Cell 103: 239-252, 2000.

31. Faris M,Kokot N,Latinis K, Kasibhatla S, Green DR, Koretzky GA and Nel A: The c-Jun N-terminal kinase cascade plays a role in stress-induced apoptosis in Jurkat cells by up-regulating Fas ligand expression. J Immunol 160: 134-144, 1998.
32. Minamino T, Christou H, Hsieh CM, Liu Y, Dhawan V, Abraham NG, Perrella MA, Minamino T, Christou H, Hsieh CM, Liu Y, Dhawan V, Abraham NG, Perrella MA, Mitsialis SA and Kourembanas S: Targeted expression of heme oxygenase-1 prevents the pulmonary inflammatory and vascular responses to hypoxia. Proc Natl Acad Sci USA 98: 8798-8803, 2001.

33. Court FA and Coleman MP: Mitochondria as a central sensor for axonal degenerative stimuli. Trends Neurosci 35: 364-372, 2012.

34. Yamaguchi R, Andreyev A, Murphy AN, Perkins GA, Ellisman MH and Newmeyer DD: Mitochondria frozen with trehalose retain a number of biological functions and preserve outer membrane integrity. Cell Death Differ 14: 616-624, 2007. 